\title{
Liderazgo de una directora en una escuela alternativa de Educación Inicial del distrito de Jesús María
}

Tejero Chávez, Carolina Cecilia

Pontificia Universidad Católica del Perú

tejero.carolina@pucp.edu.pe

http://orcid.org/0000-0002-2952-0718

\section{Resumen}

La presente investigación se centra en el liderazgo de la directora, el cual es estudiado a través de las percepciones de las docentes y la coordinadora. El objetivo general es analizar el liderazgo de una directora en una escuela alternativa de Educación Inicial del distrito de Jesús María. Los objetivos específicos que se toman en cuenta son: (i) Describir el estilo de liderazgo de la directora en una escuela alternativa de Educación Inicial del distrito de Jesús María desde las percepciones de los docentes y la coordinadora, y (ii) Comprender la importancia del estilo de liderazgo de la directora en una escuela alternativa de Educación Inicial del distrito de Jesús María. Esta investigación pertenece a un estudio cualitativo. Se considera el empleo del método de estudio de caso, con la finalidad de abordar este liderazgo ya mencionado en una escuela alternativa. El instrumento utilizado es la guía de entrevista semiestructurada, la cual permite recoger de forma detallada las percepciones de las informantes. De esta manera, la información recogida se organizó a partir del Open Coding y el Axial Coding para realizar el análisis. Finalmente, entre los resultados más resaltantes de las percepciones de las docentes y la coordinadora se valora que la directora posee un liderazgo caracterizado por su asertividad, en relación a la llegada que tiene hacia las maestras, así como la forma en que proporciona retroalimentación, considerando aspectos positivos que tiene cada uno de los actores de la escuela.

Palabras clave: Estilo de liderazgo, escuela alternativa, percepciones

\section{Leadership of a principal in an alternative school of Initial Education of the district of Jesús María}

\begin{abstract}
This research focuses on the leadership of the principal, which is studied through the perceptions of the teachers and the coordinator. The general objective is to analyze the leadership of a principal in an alternative school of Initial Education of the district of Jesús María. The specific objectives that are taken into account are: (i) Describe the leadership style of the principal in an alternative school of Initial Education of the district of Jesús María from the perceptions of the teachers and the coordinator, and (ii) Understand the importance of the leadership style of the principal in an alternative school of Initial Education of the district of Jesús María. This research belongs to a qualitative study. The use of the case study method is considered, in order to address this aforementioned leadership in an alternative school. The instrument used is the semistructured interview guide, which allows the perceptions of the informants to be collected in detail. In this way, the information collected was organized from Open Coding and Axial Coding to perform the analysis. Finally, among the most outstanding
\end{abstract}


Congreso Internacional de Gestión Educativa, 06 y 07 de noviembre de 2020.

Pontificia Universidad Católica del Perú

results of the perceptions of the teachers and the coordinator, it is valued that the director has a leadership characterized by her assertiveness, in relation to the arrival she has towards the teachers, as well as the way in which she provides feedback, considering positive aspects that each one of the actors in the school has.

Keywords: Leadership style, alternative school, perceptions

\section{Introducción}

A lo largo de los años, el liderazgo del director se ha convertido en un aspecto fundamental para las instituciones educativas. Es en ese sentido, que se ha elaborado documentación que tiene como finalidad poder orientar la gestión que se desarrolla en la escuela.

Esta documentación se despliega desde el plano internacional y nacional. En lo que se refiere al internacional, se crea el Manual de Gestión para Directores de Instituciones Educativas que se enfoca en la calidad educativa (Unesco, 2011). Ahora, en el plano nacional el documento planteado es el Marco del Buen Desempeño del Directivo que pone el foco hacia una dirección más eficaz (Minedu, 2014).

Tomando en consideración el contexto presentado es que se hace evidente reconocer la importancia del liderazgo del director definido desde un entorno en constante cambio que "exige líderes que motiven y dirijan a la organización y a sus miembros para que estos aprendan a adaptarse a los cambios" (Senge, citado por Cuevas, Díaz, Hidalgo, 2008, p.2).

De otro lado, en lo que respecta a la escuela alternativa pone énfasis en las necesidades de los alumnos (Porowski, O’ Conner y Luo, 2014). Es así que se reconoce al estudiante como el centro de este tipo de escuela.

Ahora, en la presente investigación se planteó como problema ¿Cómo es el liderazgo de una directora en una escuela alternativa de Educación Inicial del distrito de Jesús María?

Partiendo de ello, como objetivo general del estudio se consideró: Analizar el liderazgo de una directora en una escuela alternativa de Educación Inicial del distrito de Jesús María. Como objetivos específicos: Describir el estilo de liderazgo de la directora en una escuela alternativa de Educación Inicial del distrito de Jesús María desde las percepciones de los docentes y de la coordinadora, y Comprender la importancia del estilo de liderazgo de la directora en una escuela alternativa de Educación Inicial del distrito de Jesús María.

Este proyecto de investigación se enmarcó en el enfoque cualitativo, ya que analiza el liderazgo de una directora desde el contexto de una escuela alternativa. En relación al método que se utilizó fue el estudio de caso, siendo este el liderazgo de la directora. Las informantes fueron cinco maestras y la coordinadora. Y la técnica seleccionada fue la entrevista semiestructurada.

Finalmente, en lo que respecta a las conclusiones se resalta que el liderazgo de la directora se caracteriza por ser asertivo, generar confianza en la comunicación, evidenciar la participación activa de los miembros de la escuela, demostrar cercanía y acogida, y poseer una escucha que engloba comprensión y empatía por los demás. 
Congreso Internacional de Gestión Educativa, 06 y 07 de noviembre de 2020.

Pontificia Universidad Católica del Perú

\section{La escuela alternativa}

Al hacer referencia al término de escuela alternativa se alude a un tipo de educación cuya finalidad es poder acompañar al alumno para que aprenda de forma autónoma. Es de esta manera, que se pone énfasis en cubrir las necesidades que tiene cada estudiante, reconociéndolo como un ser capaz.

Ahora, en relación a las características los autores resaltan las siguientes:

a) Foco en el aspecto más interno del alumno: Como mencionan Carrión y Hernández (s/f) se educa en la conciencia y experiencia. En ese sentido es que se refleja la consideración del aspecto emocional del estudiante que incluye sus reflexiones, pensamientos, sentimientos.

b) Centro en las necesidades del estudiante: al respecto se concibe al alumno como un ser único que tiene su propio ritmo de aprendizaje y es por ello que se debe atender a sus necesidades particulares como argumentan Porowski, O’ Conner y Luo, 2014.

c) Diseño de planes de estudios flexibles: Aronson (citado por Murray y Holt, 2014) resalta la búsqueda de métodos que puedan atender las necesidades de los estudiantes. Frente a ello, es que se hace necesario encontrar las estrategias más idóneas para acompañar el aprendizaje de los alumnos, partiendo de una organización de las secuencias de clases más abierta.

d) Consideración de la personalidad del estudiante y el respeto por la diversidad: esta característica refleja lo que argumenta Frades (2016) sobre lo importante que es tomar en cuenta cómo es cada alumno. Se debe procurar un ambiente que contribuya a la reflexion.

Frente a lo que se ha descrito, se podría resaltar como característica predominante de la escuela alternativa la consideración de las necesidades de los alumnos tal como lo plantean Porowski, O’Conner y Luo (2014), y Murray y Holt (2014). A continuación, se muestran las características mencionadas por los autores:

Figura 1. Características de las escuelas alternativas

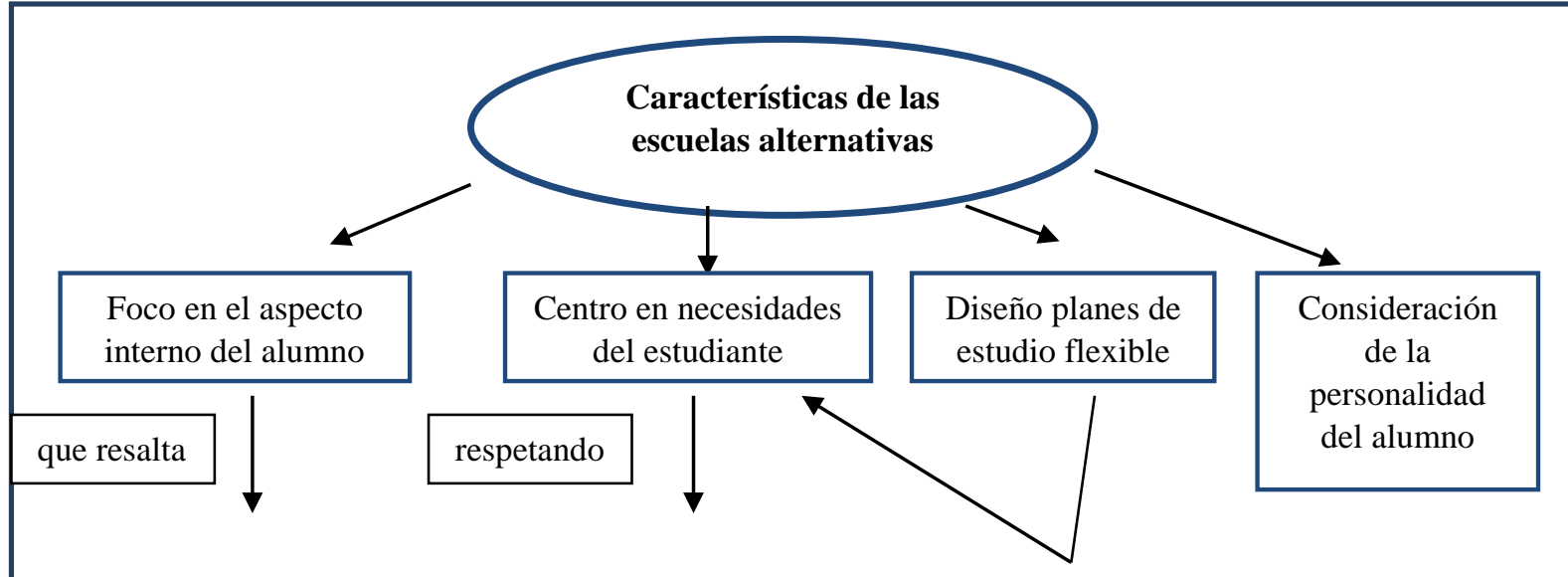


Congreso Internacional de Gestión Educativa, 06 y 07 de noviembre de 2020.

Pontificia Universidad Católica del Perú

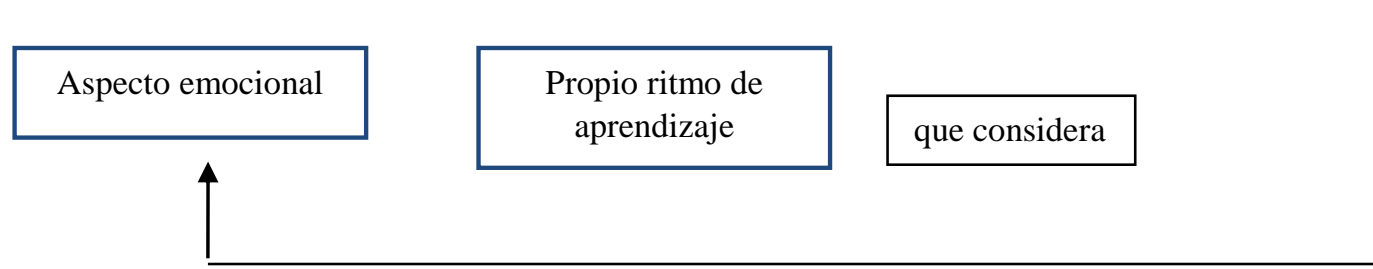

que tome en cuenta

Elaboración propia

En conclusión, se reconoce al estudiante como el centro del proceso de aprendizaje que tiene características particulares las cuales deben ser tomadas en cuenta desde el acompañamiento del maestro y el desarrollo de los planes de estudios en la escuela, que garantice la atención no sólo de los aspectos cognitivos sino también de los emocionales.

\section{EI liderazgo del director y sus estilos}

En el presente apartado se desarrollará la definición del concepto de liderazgo, así como se describirán los estilos que lo componen, tomando en cuenta sus características.

$\mathrm{Al}$ considerar el entorno cambiante en el que se contextualiza la escuela es que se hace necesario el desarrollo de un liderazgo del director que la gestione desde las demandas actuales de nuestra sociedad.

Según Chamorro (2005), se define este liderazgo como el proceso en el cual participan los actores que forman parte de la organización educativa partiendo de una mirada integrada para lograr las metas que se trazaron.

En ese sentido, como añaden Cantero y Pantoja (2016) se hace necesario también el aporte de la comunidad en relación a la organización y el funcionamiento de la escuela.

Ahora, considerando como se mencionó en líneas anteriores la participación de todos es que el liderazgo del director debe caracterizarse por motivar a todas estas personas hacia una mejora colectiva (Rodríguez, 2018). Pero, ¿cómo lograr esta mejora?

Al respecto, Calatayud (2010) propone una gestión de la escuela que contribuya con la capacidad de asumir riesgos que permitan el logro de un proyecto en común desde un entorno idóneo y sano (Vargas, 2010).

Entonces, se puede concluir que el liderazgo del director es un elemento transcendental en la gestión de la escuela desde una mirada que posibilite la mejora en conjunto, puesto que se reconoce que todos los miembros pertenecientes a la organización son una pieza clave para lograrlo.

Figura 2. EI liderazgo del director

El liderazgo del director 


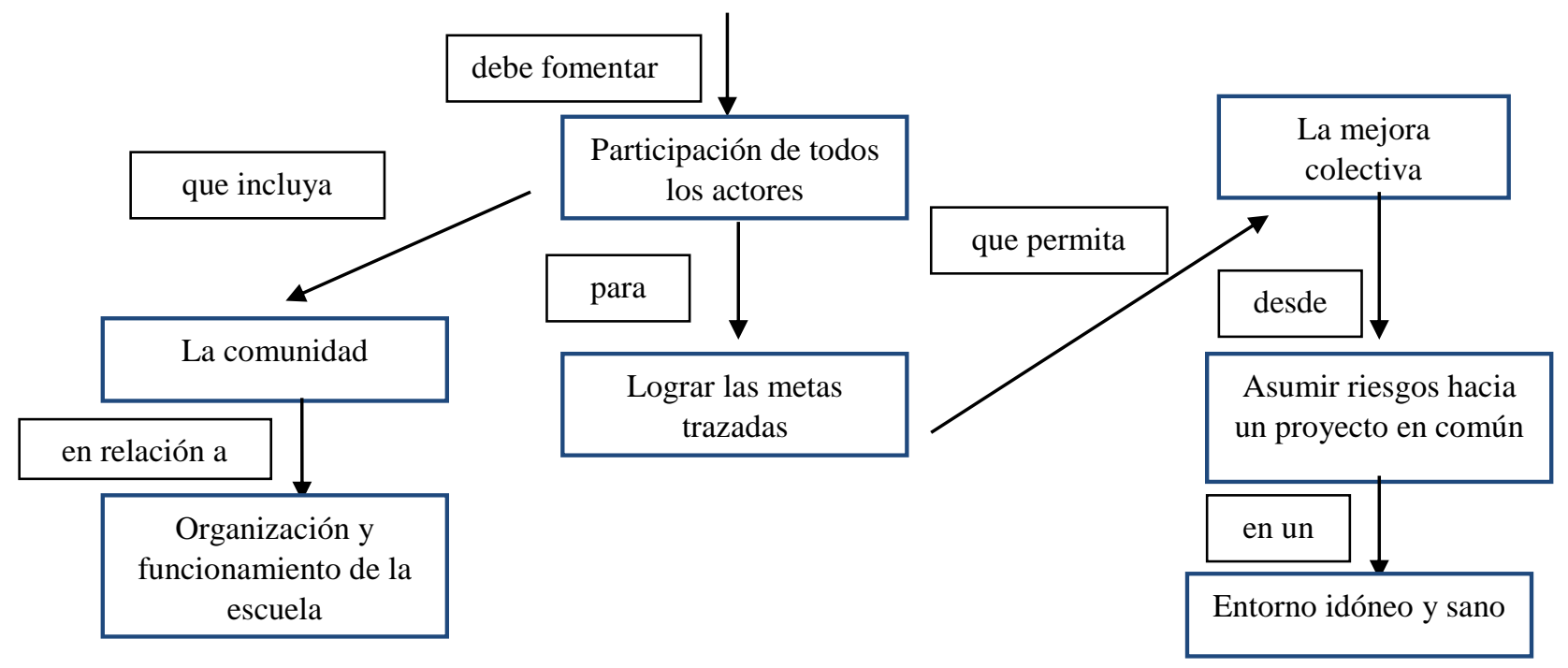

Elaboración propia

Ahora, es importante considerar el término de estilo de liderazgo que se define como "el patrón de conducta visto por terceros" (Rodríguez, Pedraja, Ganga, 2017, p. 132). De esta manera, se puede deducir que este estilo es el que condiciona cómo se ejecutará la función del director y cómo regirán su trabajo los actores de la escuela.

En relación a esta investigación, se han considerado: el liderazgo distribuido, el liderazgo transformacional, el liderazgo directivo, el liderazgo pedagógico y el liderazgo transaccional.

a) Liderazgo distribuido: $\mathrm{Al}$ respecto es necesario en este tipo de liderazgo fomentar la integración y el trabajo en grupo como mencionan Sepúlveda y Aparicio (2017). Entonces, a partir de esta dinámica se puede generar espacios de reflexión que permitan adaptarse a las exigencias del entorno cambiante.

b) Liderazgo transformacional: es importante como plantean Cabrejos y Torres (2014) que la escuela apueste por el cambio desde una comunicación que conlleve la participación de todos en las decisiones, fomentándose así el trabajo en conjunto.

c) Liderazgo directivo: se reconoce al director como un actor que debe llevar a cabo cambios en la organización educativa como argumentan Reyes, Trejo y Topete (2017), pero que además influya en el logro de metas que contribuyan a la ejecución de estos cambios.

d) Liderazgo pedagógico: este tipo de liderazgo resalta la mejora de la práctica docente (Rodríguez-Molina, 2011). En ese sentido, se realiza una revisión de los procesos de enseñanza-aprendizaje. 
Congreso Internacional de Gestión Educativa, 06 y 07 de noviembre de 2020.

Pontificia Universidad Católica del Perú

e) Liderazgo transaccional: se orienta en las negociaciones que realiza el directivo con los actores de la escuela (Peralta, Olsen, Pezzi y Sanjurjo, 2016), donde éstos últimos esperan algo a cambio.

\section{Diseño Metodológico}

El presente estudio se enmarca en el enfoque cualitativo, el cual recoge las perspectivas de los informantes a partir de una situación específica (Marshall y Rossman, citados por Iño, 2018). Este concepto tiene relación con los fines del estudio puesto que es desde las percepciones de las entrevistadas que se obtendrá información sobre el liderazgo de la directora.

Por otro lado, la investigación se considera de carácter descriptivo, ya que se enfoca en resaltar las características de un fenómeno como plantean Verd y Lozares, 2016. El contexto elegido para este estudio fue el de la escuela alternativa.

Ahora, al pertenecer al enfoque cualitativo es que el método seleccionado fue el estudio de caso. Éste como menciona Stake (1998) se enfoca en el estudio de un caso complejo y particular. Por este motivo, es que en la investigación se define al liderazgo de la directora desde su particularidad y en relación al contexto en el que está inmerso.

\section{Problema de investigación}

¿Cómo es el liderazgo de una directora en una escuela alternativa de Educación Inicial en el distrito de Jesús María?

\section{Objetivos de la investigación}

\section{Objetivo general}

Analizar el liderazgo de una directora en una escuela alternativa de Educación Inicial en el distrito de Jesús María

\section{Objetivos específicos}

Describir el estilo de liderazgo de la directora en una escuela alternativa de Educación Inicial en el distrito de Jesús María desde las percepciones de los docentes y la coordinadora.

Comprender la importancia del estilo de liderazgo de la directora en una escuela alternativa de Educación Inicial del distrito de Jesús María

\section{Categorías y subcategorías de la investigación}

Para el presente estudio es que se consideró como categoría el estilo de liderazgo de la directora. De ésta es que se desprenden las siguientes subcategorías: liderazgo distribuido, liderazgo transformacional, liderazgo directivo, liderazgo pedagógico y liderazgo transaccional. 
Congreso Internacional de Gestión Educativa, 06 y 07 de noviembre de 2020.

Pontificia Universidad Católica del Perú

\section{Técnica e instrumento de recojo de información}

Para fines del presente estudio se tomó en cuenta la utilización de la técnica de la entrevista semiestructurada. Este tipo de entrevista se caracteriza como plantean Hammer y Wildavsky (1990) por la realización de preguntas abiertas que permiten que el entrevistador pueda desarrollarlas según su criterio.

En cuanto al instrumento se elaboró una guía de entrevista semiestructurada que contenía preguntas relacionadas a las categorías y que posibilitaban repreguntar con el fin de recabar la mayor parte de información desde las percepciones de los informantes.

\section{Los informantes}

Esta investigación cuenta con una muestra de cinco maestras de un total de catorce docentes en la escuela, y la coordinadora.

\section{Procedimiento para el análisis e interpretación de resultados}

Para el desarrollo del análisis se elaboró una matriz que especificaba por pregunta los hallazgos que fueron encontrados para cada una de las entrevistadas. Seguidamente, se organizó en otra matriz de análisis, conocida como el open coding, la integración de los hallazgos reconocidos como elementos emergentes.

De esta manera, se realizó una codificación que permitió la interpretación de los resultados en relación a las categorías del estudio y posteriormente, se llevó a cabo la relación existente entre los conceptos en el análisis (axial coding).

\section{Resultados}

a) Liderazgo distribuido: se resalta según las informantes la participación de todos los actores de la escuela "siempre hay un tiempo para cada uno de los actores, si quieres decir algo lo puedes hacer en el momento" (D2). En este sentido, se evidencia cómo la directora promueve espacios para que cada uno desde su rol pueda dar a conocer sus ideas, sobre todo en el contexto de las reuniones de staff donde participa todo el equipo, pero también durante la cotidianidad. Este aspecto se relaciona con lo mencionado por Sepúlveda y Aparicio (2017), sobre la promoción del trabajo en grupo desde la posibilidad de expresar su opinión, considerando que todo lo mencionado es de relevancia para toda la comunidad educativa.

b) Liderazgo transformacional: las entrevistadas recalcan la búsqueda de soluciones en conjunto "de todas maneras porque sino ella no te incluiría en los planes de mejora siempre está como que busca soluciones, pero en conjunto no solamente ella" (D3). Las líneas anteriores reflejan cómo la directora se preocupa por involucrar a los miembros de la escuela hacia un proyecto en común desde la participación activa que tienen para sugerir soluciones con miras a lograr cambios positivos en la escuela. Al respecto, ello coincide con lo que señalan Cabrejos y Torres (2014), resaltando el intercambio desde la mirada de equipo y 
la comunicación que se fomenta con todos los miembros de la comunidad educativa en torno a aspectos que competen a todos.

c) Liderazgo directivo: las informantes hacen evidente la toma de decisiones de manera autónoma en los salones de clase "entonces me dio la confianza, porque al inicio como que no quería mover nada no me atrevía a mover ningún mueble, y me dijo atrévete a hacerlo, entonces siento que ella te permite cambiarlo y te apoya" (D1). Lo mencionado anteriormente, refleja cómo la directora incentiva y brinda al equipo docente la libertad para ejecutar cambios en sus clases, desde un ambiente de confianza que de alguna forma las empodera y da soporte si fuese necesario. Ello se relaciona con lo que postulan Reyes, Trejo y Topete (2017) sobre la generación de cambios por parte del directivo, pero tomando en consideración los aportes de los miembros de la comunidad educativa.

d) Liderazgo pedagógico: al respecto las entrevistadas mencionan su participación en diplomados y cursos con la finalidad de compartir sus aprendizajes "la directora pregunta ¿qué van hacer el siguiente año?, entonces una maestra que desea continuar, seguir aprendiendo muchísimo más te facilita, qué te parece si llevas este diplomado para que te puedas empoderar más de esta filosofía y quizás puedas aterrizar más, entonces te dan como que esa oportunidad" (D3). Considerando lo descrito, la directora muestra interés por brindar continuamente posibilidades para que el equipo docente se capacite y mejore su práctica, desde una mirada de mejora para la escuela pero también para sí mismas. Este aspecto coincide con lo que postula Rodríguez-Molina (2011) sobre la preocupación de la directora en la mejora de la práctica docente en un contexto en el que se promueve el aprendizaje constante y la posibilidad de compartirlo con todos.

e) Liderazgo transaccional: las informantes señalan la promoción de feedback con el propósito de compartir estrategias "siempre ha habido como apertura de poder, conversar con la otra compañera sobre estrategias que a ella le están funcionando y tú aún no lo estás implementando que quizá ni se te ocurría hacerlo" (D5). Al respecto, se puede deducir cómo la directora promueve en la comunidad educativa un diálogo abierto que permite compartir diversas ideas que contribuyan a mejorar el trabajo. De esta manera, se refleja un ambiente que promueve una comunicación cercana y enriquecedora desde una mirada colectiva que puede beneficiar a todos. Ello se relaciona con lo planteado por Peralta, Olsen, Pezzi, Sanjurjo (2016) sobre el intercambio de relaciones, que conlleva a la creación de una escuela que mejora en conjunto.

\section{Conclusiones}

1. El estudio realizado evidencia aspectos necesarios en la escuela alternativa en relación a su gestión. A partir de ello, las características de la directora deberían enfocarse en: promover el intercambio, buscar estrategias desde la reflexión y 
Congreso Internacional de Gestión Educativa, 06 y 07 de noviembre de 2020.

Pontificia Universidad Católica del Perú

poseer asertividad. Ello influirá en el estilo de liderazgo de la directora en la organización educativa.

2. En relación al estilo de liderazgo distribuido, se resalta la participación de todos los actores de la escuela en la expresión de opiniones en las reuniones. Ello permite el desarrollo del sentido de comunidad.

3. En el estilo de liderazgo transformacional, se hace evidente la promoción del trabajo en equipo por parte de la directora, permitiendo el intercambio entre docentes desde sus experiencias.

4. Considerando el estilo de liderazgo directivo, se hace mención al rol que tiene la directora como una persona que genera cambios en la escuela. Este aspecto se lleva a cabo desde la autonomía que brinda a las docentes para realizar cambios en sus aulas.

5. En relación al estilo de liderazgo pedagógico, las informantes resaltan la promoción del mejoramiento de la práctica docente a través de capacitaciones y el feedback que se proporciona en las supervisiones participativas en el aula.

6. Finalmente, en el estilo de liderazgo transaccional se hace hincapié en los cambios que ejecuta la directora, siendo un ejemplo a seguir en todos los momentos que forman parte de la cotidianidad de la escuela.

\section{Referencias Bibliográficas}

Cabrejos, H., Torres, L. (2014). El liderazgo transformacional como apoyo en la gestión pedagógica del director. Revista de Investigación y Cultura UCV-HACER, 3 (2), 10-16. Recuperado de http://revistas.ucv.edu.pe/index.php/UCVHACER/article/view/716

Calatayud, M. (2010). El director que se necesita hoy en las organizaciones escolares. Revista Padres y Maestros, (334), 5-9. Recuperado de https://www.google.com/search?q=El+director+que+se+necesita+hoy+en+las+orga nizaciones+escolares.\&rlz=1C1CHBF_esPE797PE797\&oq=El+director+que+se+n ecesita+hoy+en+las+organizaciones+escolares.\&aqs=chrome..69i57j0.30707j0j4\& sourceid $=$ chrome $\&$ ie $=$ UTF- $8 \#$

Cantero, N., Pantoja, A. (2016). El papel del director de un centro transformado en comunidad de aprendizaje desde la perspectiva del profesorado. Revista Aula $\begin{array}{lllll}\text { encuentro, } & 2 & \text { (18), } & \text { 76-96. }\end{array}$ https://dialnet.unirioja.es/servlet/articulo?codigo $=5753056$

Carrión, E., Hernández, M. (s/f). Las escuelas alternativas. Una aproximación a la educación del futuro. Modelos educativos para el siglo XXI. Escuelas de calidad, especialización excesiva educación por competencias. Primer congreso online internacional sobre Desigualdad Social y Educativa del Siglo XXI, Murcia. Recuperado de http://www.eumed.net/libros-gratis/actas/2016/desigualdad/34.pdf

Chamorro, D. (2005). Factores determinantes del estilo de liderazgo del director-a (tesis de posgrado). Universidad Complutense de Madrid. Recuperado de https://dialnet.unirioja.es/servlet/tesis?codigo $=17240$ 
Congreso Internacional de Gestión Educativa, 06 y 07 de noviembre de 2020.

Pontificia Universidad Católica del Perú

Cuevas, M., Díaz, F., Hidalgo, V. (2008). Liderazgo de los directores y calidad de la educación. Un estudio del perfil de los directivos en un contexto pluricultural. Revista de currículo y formación del profesora, 12 (2), 1-20. Recuperado de https://www.redalyc.org/articulo.oa?id=56712211

Frades, E. (2016). La renovación pedagógica en España: un movimiento social más allá del Didactismo. Revista Tendencias pedagógicas, (27), 259-284. Recuperado de https://revistas.uam.es/tendenciaspedagogicas/article/view/3014/3228

Hammer, D y Wildavsky, A. (1990). La entrevista semi-estructurada de final abierto: aproximación a una guía operativa Revista Universitat de Barcelona, (4), 23-61. Recuperado https://www.jstor.org/stable/27753290?seq=1\#page_scan_tab_contents

de:

Iño, W. (2018). Investigación educativa desde un enfoque cualitativo: la historia oral como método Revista Voces de la educación 3 (6), 93-110. Recuperado de: https://www.revista.vocesdelaeducacion.com.mx/index.php/voces/article/view/1 23

Ministerio de Educación. (2014). Marco de Buen Desempeño del Directivo. Recuperado de: http://www.minedu.gob.pe/n/xtras/marco_buen_desempeno_directivo.pdf

Murray, B., Holt, C. (2014). Alternative Education Completers: A Phenomenological Study. Revista NCPEA, 1 (1), 185-203. Recuperado de https://files.eric.ed.gov/fulltext/EJ1105735.pdf

Peralta, Y., Olsen, C., Pezzi, L., Sanjurjo, N. (2016). Liderazgo transaccional y transformacional de voluntarios jóvenes y adultos del Mar del Plata. Revista $\begin{array}{lllll}\text { Psicoperspectivas, } & 15 & \text { (3), } & \text { 145-156. Recuperado de }\end{array}$ http://web.a.ebscohost.com.ezproxybib.pucp.edu.pe:2048/ehost/pdfviewer/pdfvi ewer?vid=1\&sid=6c11c11a-68aa-41aa-aaf4-513b94b03943\%40sessionmgr4006

Porowski, A., O’Conner, R., Luo, J. (2014). How do states define alternative education? National Center for Education Evaluation and Regional Assistance, 1-18. Recuperado de_https://files.eric.ed.gov/fulltext/ED546775.pdf

Reyes, V., Trejo, M., Topete, C. (2017). El liderazgo directivo y la gestión en el nivel medio superior del Instituto Politécnico Nacional de México: una mirada desde los estudiantes. Revista iberoamericana para la investigación y el desarrollo educativo, 8 (15), 1-35. Recuperado de http://www.scielo.org.mx/scielo.php?script=sci_arttext\&pid=S2007$74672017000200081 \& \operatorname{lng}=$ es\&nrm=iso

Rodríguez, E., Pedraja, L., Ganga, F. (2017). La relación entre los estilos de liderazgo y el desempeño de los equipos de dirección intermedia. Revista Contabilidad y Negocios, $12 \quad$ (23), 129-144. Recuperado de http://www.redalyc.org/pdf/2816/281653630010.pdf

Rodríguez, E. (2018). El ejercicio de la función directiva en contextos complejos: su profesionalización. Revista Perspectiva Educacional, 57 (3), 131-152. 
Congreso Internacional de Gestión Educativa, 06 y 07 de noviembre de 2020.

Pontificia Universidad Católica del Perú

Recuperado

de

https://scielo.conicyt.cl/scielo.php?script=sci_arttext\&pid=S0718-

97292018000300131

Rodríguez-Molina, G. (2011). Funciones y rasgos del liderazgo pedagógico en los centros de enseñanza. Revista Educación y educadores, 14 (2), 253-267. Recuperado de http://www.scielo.org.co/pdf/eded/v14n2/v14n2a02.pdf

Sepúlveda, F., Aparicio, C. (2017). El desafío de los directores de escuelas chilenas: Liderando a partir de un enfoque instruccional a un enfoque distribuido. Revista Gestión de la Educación, 7 (2), 1-19. Recuperado de https://revistas.ucr.ac.cr/index.php/gestedu/article/view/30599

Stake, R. E. (1998). Investigación con estudio de casos. Madrid: Ediciones Morata

Unesco. (2011). Manual de Gestión para Directores de Instituciones Educativas. Recuperado de: https://unesdoc.unesco.org/ark:/48223/pf0000219162

Vargas, I. (2010). ¿Por qué es esencial discutir acerca del liderazgo en la gestión escolar? Revista Electrónica Educare, 14 (1), 59-66. Recuperado de https://dialnet.unirioja.es/descarga/articulo/4780955.pdf

Verd, J y Lozares, C. (2016). Introducción a la investigación cualitativa. Fases, métodos y técnicas. Madrid: Editorial Síntesis 